\title{
Suitability of the Decentralised Wastewater Treatment Effluent for Agricultural Use: Decision Support System Approach
}

\author{
William Musazura * and Alfred O. Odindo
}

check for updates

Citation: Musazura, W.; Odindo, A.O. Suitability of the Decentralised Wastewater Treatment Effluent for Agricultural Use: Decision Support System Approach. Water 2021, 13, 2454. https://doi.org/10.3390/ w13182454

Academic Editor: Panagiotis Karanis

Received: 14 July 2021

Accepted: 2 September 2021

Published: 7 September 2021

Publisher's Note: MDPI stays neutral with regard to jurisdictional claims in published maps and institutional affiliations.

Copyright: (c) 2021 by the authors. Licensee MDPI, Basel, Switzerland. This article is an open access article distributed under the terms and conditions of the Creative Commons Attribution (CC BY) license (https:// creativecommons.org/licenses/by/ $4.0 /)$.
Discipline of Crop Science, School of Agricultural, Earth and Environmental Sciences, University of KwaZulu-Natal, Private Bag X01, Scottsville, Pietermaritzburg 3209, South Africa; Odindoa@ukzn.ac.za

* Correspondence: wmusazura@gmail.com; Tel.: +27-742935867

\begin{abstract}
The decentralised wastewater treatment system (DEWATS) is an onsite sanitation technology that can be used in areas away from municipal sewerage networks. The discharge of effluent emanating from DEWATS into water bodies may cause pollution. Agricultural use of the effluent may improve crop yields and quality thereby contributing to food security in low-income communities. There are drawbacks to the agricultural use of treated wastewater. Therefore, the study assessed the crop, environmental and health risks when irrigating with anaerobic filter (AF) effluent using the Decision Support System (DSS) of the South African Water Quality Guideline model, in four South African agroecological regions, three soil types, two irrigation systems and three different crops. The model was parameterised using AF effluent characterisation data and simulated for 45 years. The model predicted that there are no negative impacts for using AF effluent on soil quality parameters (root zone salinity, soil permeability and oxidisable carbon loading), leaf scorching and irrigation equipment. The problems were reported for nutrient loading ( $\mathrm{N}$ and $\mathrm{P}$ ) in maize and microbial contamination in cabbage and lettuce. It was recommended that the effluent should be diluted when used for maize production and advanced treatment should be explored to allow unrestricted agricultural use.
\end{abstract}

Keywords: human excreta materials; nutrient recovery; treated wastewater; wastewater reuse; water quality guideline

\section{Introduction}

Global water supply is threatened by population dynamics, characterised by excessive urbanisation. Currently, in South Africa, about $63 \%$ of the people are living in urban areas and this figure is expected to reach $71 \%$ by the year 2030 [1]. This is straining on municipal service delivery as they are failing to provide adequate housing, sanitation and clean water. Most of the migrants are residing in informal settlements away from the municipal sewerage network, and in addition, they are unemployed and food insecure [2]. However, connecting people to centralised sanitation systems is difficult especially in areas with undulating terrain and where unplanned settlements are continuously emerging. Therefore, the Decentralised Wastewater Treatment System (DEWATS) can be a potential sanitation solution in such areas. The DEWATS is a modular system that comprises of the settler, anaerobic baffled reactor (ABR), anaerobic filter (AF) and planted gravel filters (PGFs). Solids are settled down in the settler and the suspended scum is removed. The wastewater moves through the ABR, where anaerobic degradation of organic compounds occurs, and this is later polished in the anaerobic filter. The resulting effluent (AF effluent) contains mineral nutrients and some pathogens, hence it is further treated PGFs, which comprises of the vertical flow constructed wetlands (VFCW) and the horizontal flow constructed wetlands (HFCW) [3]. The final DEWATS effluent may not meet the stringent South African discharge quality. Therefore, discharging it into water bodies may cause pollution, evidenced by algal blooms, death of aquatic life and sometimes expose people 
to waterborne diseases. However, to ensure sustainability the development of sanitation systems should be linked to agriculture in a way that solves socio-economic challenges in low-income communities while protecting the environment.

The DEWATS effluent is a potential agricultural resource that can be used for agriculture as a source of water and nutrients under different agricultural systems; field and hydroponic systems [4]. Studies have confirmed its ability to improve soil properties [5] and crop yields [6]. Just like other domestic wastewater, the DEWATS effluent is low in concentrations of heavy metals [7]. However, there are other long term potential limitations to the agricultural use of treated wastewater, which should be assessed with regards to DEWATS effluent. These include effects on soil properties, crop response to salinity, microbial risks and heavy metal accumulation with long term irrigation ( $>200$ years) [8-10].

Irrigation water quality parameters such as the concentration of $\mathrm{Cl}^{-}, \mathrm{B}$, atrazine, microorganisms and macronutrients (NPK) can have direct and indirect impacts on soil quality (environment), crop yields and quality and human health [11]. The amount of effluent to be applied, its effects on crop, nutrient loading and potential microbial hazards depend on irrigation management practices, water quality, climate, soil type and crop type determine [12]. du Plessis et al. [12] developed a risk-based, site-specific irrigation water quality guideline based on the Department of Water and Sanitation [11] generic guideline, and the latest local and international guidelines. The tool was developed in the form of a Decision Support System (DSS) to comply with the latest requirements of the Department of Water and Sanitation [13] South African National Water Act of 1998. The DSS is a novel tool to assess the suitability of water of a certain quality for agricultural use and can be used for any wastewater such as the DEWATS effluent (AF effluent). Therefore, this study aimed to assess the crop, environmental and health risks associated with irrigation using AF effluent using the DSS model. The specific objectives were to (i) assess the suitability of AF effluent irrigation water quality for different crops (maize, cabbage and lettuce), soils (coarse sand, sandy loam and clay) in four agro-ecological regions of South Africa, with a special focus on impacts on microbial contamination, crop quality, impacts on irrigation infrastructure, soil quality and environmental pollution, and (ii) provide recommendations for optimising soil quality, crop yield, minimise human health and prevent irrigation equipment damage when AF effluent is used for irrigation across agro-ecological regions of South Africa.

\section{Materials and Methods}

\subsection{Description of the Decision Support System}

The South African Department of Water and Forestry water quality guidelines of 1996 [13] was produced by a panel of experts following national and international guidelines. The guideline was developed based on the Food and Agriculture Organization (FAO) water quality guidelines of agricultural importance [10,14], World Health Organization (WHO) parameters of health significance [15,16], the United States Environmental Protection Agency (USEPA) parameters of environmental importance [17] and other international guidelines. As knowledge was gained and practices changed with time, the South African Water Quality Guideline (SAWQG) was developed in 2017 to include developments not addressed in the Department of Water and Sanitation [11] guidelines. The guideline considers risk-based and site-specific approaches in compliance with the Department of Water and Sanitation [13] revised general authorisation for wastewater use in agriculture.

A schematic diagram of the DSS is shown in Figure 1. It consists of two major components: the assessment of water quality for agricultural use and the water quality requirements for a specific use. The DSS follows an integrated approach, using the Lazarus computer code linking input data, calculation procedures and databases to produce output on irrigation water quality guidelines [12]. 

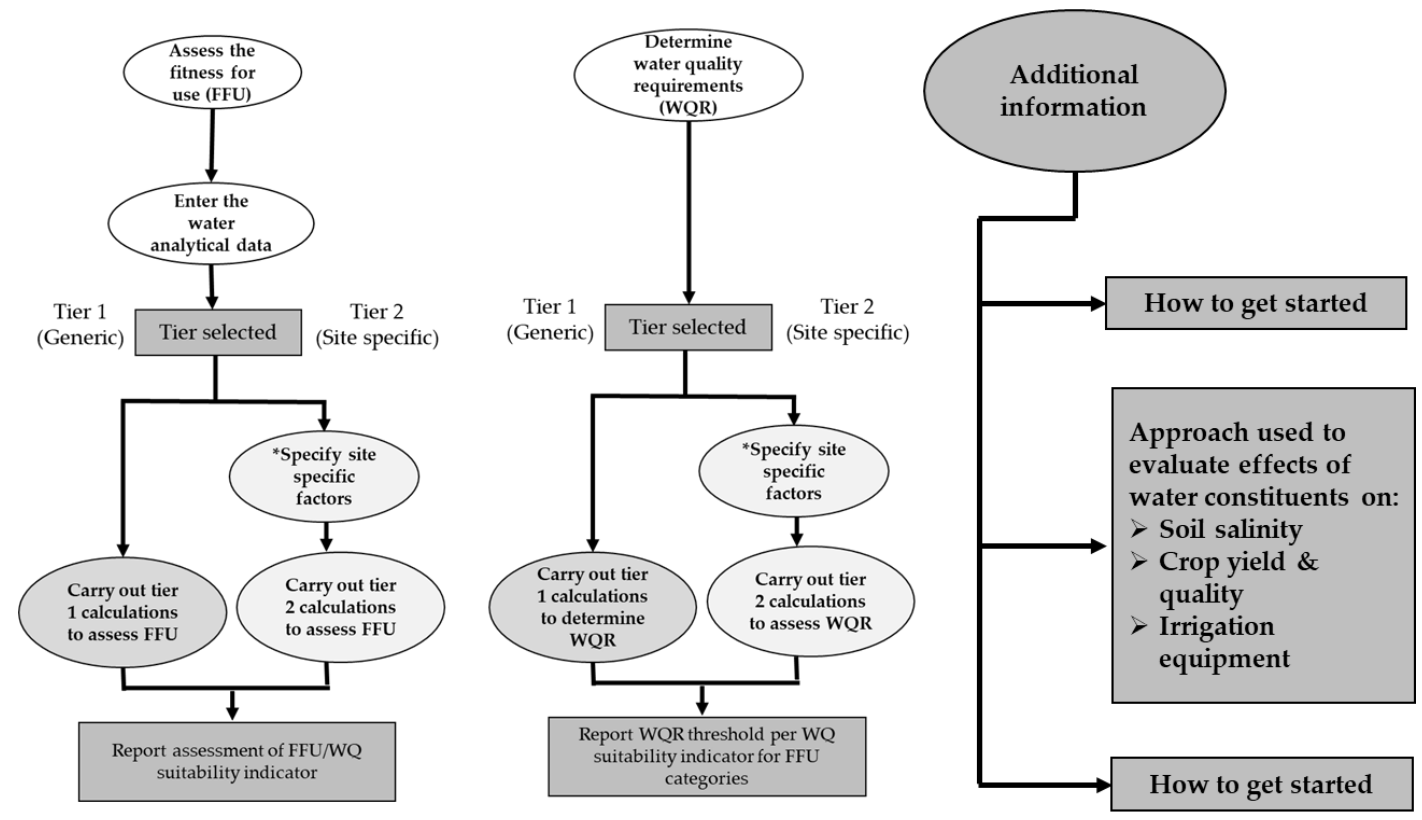

${ }^{*}$ Crop/climate/Soil/Irrigation management

WQ; water quality

FFU; Fitness for use

Figure 1. The structure of the risk-based, site-specific Decision Support System (DSS). Adapted and modified from du Plessis et al. [12].

According to du Plessis et al. [12], tier number 1 calculates the interaction of water components, crop and soil water uptake. The soil-water-crop interaction considers a 4-layer soil with an assumption that $40 \%$ of the crop water requirements are extracted from the top layer followed by the second layer (30\%), the third layer $(20 \%)$ and $10 \%$ from the bottom layer. The model calculates the steady-state concentration of the solution in each layer from the characteristics of the irrigation water and the leaching profile of the whole profile. An assumption is made that a leaching fraction of 0.1 prevails in the soil and there are no allowances made for rain. As a result, the calculated output for evaluating the fitness for use (FFU) for a specific water type and the water quality requirement (WQR) are always the same.

The tier 2 calculations are done using the modified SWB model [12]. This is done to simulate the interaction between water quality, climate, and soil and crop type on water balance, soil quality, crop yield and quality, the concentration of trace elements, irrigation equipment and microbial risks.

Water fluxes are simulated following a cascading approach (literally known as the tipping bucket method); when each layer reaches the soil water saturation point, the water 'tips' to the next layer [18]. The soil component of the SWB model divides the soil into 11 different layers and the soil physical properties of volumetric permanent wilting point, field capacity and bulk densities are specified for each layer [18]. The texture of each layer is predetermined, and the default drainage parameters (drainage fraction and drainage rate) are available in the soil subcomponent. The effects of salinity on yield are estimated from electrical conductivity (EC) values calculated for each layer and averaged for the whole profile. The model allows the user to run simulations over several seasons (up to 45 years) to increase the accuracy of the results.

The crop management component is included, and the user must select irrigation management options such as irrigation system (surface vs. sprinkler), irrigation timing (percentage soil moisture depletion, irrigation intervals in days or a fixed amount in $\mathrm{mm}$ ) and the refill options (room for rainfall, field capacity, leaching requirement or fixed amount). 
Wastewater contains elements that are required by plants, but some are toxic and significantly affect crop yield. Specific ions of concern include $\mathrm{B}, \mathrm{Na}^{+}$and $\mathrm{Cl}^{-}$which are present in some treated wastewaters. These ions are taken up by the crop through the transpiration stream, accumulate in the leaf tissues of sensitive crops and after exceeding certain thresholds kill the leaf tissues. Alternatively, the specific ion can be adsorbed through wetted foliage especially when sprinkler irrigation is used. The DSS is thus able to estimate yield, considering the impacts of root zone salinity and crop toxicity using Equation (1) [12]:

$$
\mathrm{Y}(\text { yield })=100-\mathrm{b}(\mathrm{RZC}-\alpha)
$$

where: $\mathrm{b}$ is the slope of the yield response curve exceeding the threshold concentration. RZC is the root zone concentration of the constituent of concern and $\alpha$ is the threshold concentration of the element of concern.

The three important macronutrients which have significant effects on crop yield are N, P and K [19]. Treated wastewater contains macronutrients required by crops, hence its use in agriculture helps to minimise fertiliser costs [20]. However, high concentrations of nutrients have direct and indirect drawbacks. Excessive amounts of $\mathrm{N}$ cause delayed maturity and uneven ripening in flowering plants. Nitrogen and P may cause non-point source pollution. Nitrate can leach into groundwater resources [21] and runoff losses of phosphorus into nearby rivers can cause eutrophication [22]. Potassium is a less toxic cation that does not have any environmental impacts. The DSS calculates the N, P and K loading and removal by plants. The model assesses the suitability of the water quality component for use based on the percentage of the elements removed by plant uptake per amount of nutrients applied. The removal of $10 \%$ of the $\mathrm{N}, \mathrm{P}$ and $\mathrm{K}$ from wastewater by plants is categorised as ideal, $10-30 \%$ acceptable, $30-50 \%$ tolerable and $>50 \%$ unacceptable [12].

\subsection{Model Parameterisation}

\subsubsection{Study Sites}

Irrigation water of certain quality affects soil and crop quality differently due to differences in irrigation management practices and soil properties in various agroecological regions. Four different study sites belonging to different climatic regions classified according to Köppen-Geiger classification system [23] were selected and are described in Table 1.

Table 1. The selected four agroecological regions of South Africa are classified according to the Köppen-Geiger classification system [23].

\begin{tabular}{|c|c|c|c|c|}
\hline $\begin{array}{l}\text { Climatic } \\
\text { Region }\end{array}$ & Place & Coordinates & $\begin{array}{l}\text { Altitude } \\
\text { (masl) }\end{array}$ & Description \\
\hline \multirow{3}{*}{1} & Pretoria & $-25.7500 \mathrm{~S} ; 28.26670 \mathrm{E}$ & 1360 & \multirow{3}{*}{$\begin{array}{c}\text { Cwb; Warm } \\
\text { temperate, Dry winter, } \\
\text { Warm summer }\end{array}$} \\
\hline & Roodeplaat & $-25.6000 \mathrm{~S} ; 28.35000 \mathrm{E}$ & 1240 & \\
\hline & Servontein & $-29.7500 \mathrm{~S} ; 30.13333 \mathrm{E}$ & 1440 & \\
\hline \multirow{3}{*}{2} & Messina & $-22.2333 \mathrm{~S} ; 29.91667 \mathrm{E}$ & 500 & \multirow{3}{*}{ Bsh; Steppe, Hot Arid } \\
\hline & Pieterzburg & $-23.8667 \mathrm{~S} ; 29.45000 \mathrm{E}$ & 1250 & \\
\hline & Zebediela & $-22.233300 \mathrm{~S}, 29.916670 \mathrm{E}$ & 500 & \\
\hline \multirow{3}{*}{3} & Douglas & $-29.0500 \mathrm{~S} ; 23.76667 \mathrm{E}$ & 1024 & \multirow{3}{*}{ Bwh; Desert, Hot Arid } \\
\hline & Taung & $-27.5500 \mathrm{~S} ; 24.76667 \mathrm{E}$ & 1110 & \\
\hline & Upington & $-28.4500 \mathrm{~S} ; 21.25000 \mathrm{E}$ & 775 & \\
\hline \multirow{3}{*}{4} & Citrusdale & $-32.5667 \mathrm{~S} ; 18.98330 \mathrm{E}$ & 234 & \multirow{3}{*}{$\begin{array}{c}\text { Bsk; Steppe, Cold } \\
\text { Arid }\end{array}$} \\
\hline & Ladysmith & $-33.4833 \mathrm{~S} ; 21.03333 \mathrm{E}$ & 384 & \\
\hline & Riversdale & $-34.100000 \mathrm{~S} ; 21.266700 \mathrm{E}$ & 104 & \\
\hline
\end{tabular}

Masl; Metres above sea level. 


\subsubsection{DEWATS Effluent Characteristics}

The AF effluent biological and physicochemical properties to parameterise the DSS were obtained from characterisation data collected from the Newlands Mashu research site in Durban.

\subsubsection{Soil Types}

Soils of different textures have different physical and chemical properties which influence inter alia soil moisture retention, microbiological processes and water fluxes. Spatial variations in soil types have impacts on the extent to which irrigation water of certain quality positively or negatively affects soil and crop quality [5]. Therefore, three different soil texture types were selected for simulations and their physical properties obtained from the DSS are given in Table 2.

Table 2. Physical properties of the four contrasting soils used during the study [12].

\begin{tabular}{cccc}
\hline & Sandy Loam & Coarse Sand & Clay \\
\hline Initial salt content & Low & Low & Low \\
Profile available water $(\mathrm{mm})$ & 120 & 40 & 150 \\
Volumetric water content at field capacity $\left(\mathrm{m} \mathrm{m}^{-1}\right)$ & 0.22 & 0.08 & 0.33 \\
Volumetric water content at permanent wilting & 0.1 & 0.04 & 0.18 \\
point $\left(\mathrm{m} \mathrm{m}^{-1}\right)$ & 1.4 & 1.7 & 1.2 \\
\hline Bulk density $\left(\mathrm{g} \mathrm{cm}^{-3}\right)$ &
\end{tabular}

\subsubsection{Crop Type and Management Practices}

The three different summer crops selected were maize (Zea mays L.), lettuce (Lactuca sativa) and cabbage (Brassica oleracea var capitata). The crops were selected based on low microbial contamination risks in treated wastewater irrigation as per the WHO specifications [10]; maize has husks and the cob is produced away from the ground, cabbage is a commonly grown crop in South Africa and lettuce is the riskiest vegetable to produce.

Different crops have different climatic requirements and South Africa is generally a subtropical country that experiences seasonal variations across the year, hence a crop rotation system of a summer and a winter crop was chosen. Two irrigation systems chosen were surface and overheard irrigation to compare their impacts, especially on microbial contamination risks. The irrigation timing was based on soil moisture depletion levels.

\subsection{DSS Model Simulations}

The parameterised DSS tier 2 was simulated for a period of 45 years. The output data on FFU was recorded for the AF effluent. Its impacts on soil quality (root zone salinity, soil permeability, oxidisable $\mathrm{C}$ loading and trace element accumulation), crop yield and quality (root zone effects, leaf scorching when wetted, and crop and microbial contamination risks) and FFU of the irrigation equipment were assessed.

The percentage of time that soil root zone salinity, soil permeability (surface infiltrability and soil hydraulic conductivity) and oxidisable C (COD) loading were predicted to fall within a certain category of FFU was determined. The accumulation of trace elements was assessed as the number of years in which a certain predicted irrigation amount elevated them to threshold levels in the topsoil ( $0.3 \mathrm{~m}$ depth).

The microbial risk assessment was done to predict excess infections per 1000 persons per annum. However, atrazine damage was not assessed since it is absent in the AF effluent.

\subsection{Data Analysis}

The GenStat 21st Edition [24] was used to analyse all the quantitative and qualitative data. Qualitative data on the suitability of AF effluent for agricultural use was summarised using descriptive statistical methods. Quantitative data on nutrient uptake was subjected to analysis of variance (ANOVA). Where significant differences were reported, Bonferroni's test was done to separate differences between means. 
The crop yield and quality for maize, cabbage and lettuce were then assessed using the criteria shown in Table 3 . The percentage of time that root zone effects (salinity, B, $\mathrm{Na}^{+}$ and $\mathrm{Cl}^{-}$) fell within a certain category was assessed. The degree of leaf scorching due to $\mathrm{Na}^{+}$and $\mathrm{Cl}^{-}$was assessed qualitatively. The contribution of irrigation water to $\mathrm{N}, \mathrm{P}$ and $\mathrm{K}$ removal, directly or indirectly, was determined as a percentage of the time their removal at harvest was within FFU categories, taking into consideration the impacts of high nutrient concentrations. The total mean applied N, P and K through irrigation at harvest was also calculated and reported quantitatively.

There are four categories for assessing fitness for use and these are ideal, acceptable, tolerable and unacceptable. Therefore, in this study, it was assumed that the good water quality for a specific purpose is when at least $>50 \%$ of the time for fitness for use fall within at least the tolerable category.

Table 3. Crop yield and quality, soil quality, impacts on irrigation equipment and with a specific amount of irrigation (predicted by the DSS) [12].

\begin{tabular}{|c|c|c|}
\hline & Fitness for Use & Range \\
\hline \multicolumn{3}{|c|}{ Crop yield and quality } \\
\hline \multirow{4}{*}{$\begin{array}{l}\text { Root zone effects } \\
\text { (Relative crop yield in \%) }\end{array}$} & Ideal & $90-100 \%$ \\
\hline & Acceptable & $80-90 \%$ \\
\hline & Tolerable & $70-80 \%$ \\
\hline & Unacceptable & $<70 \%$ \\
\hline \multirow{4}{*}{$\begin{array}{l}\text { Leaf scorching when wetted } \\
\text { (Degree of leaf scorching) }\end{array}$} & Ideal & None \\
\hline & Acceptable & Slight \\
\hline & Tolerable & Moderate \\
\hline & Unacceptable & Severe \\
\hline \multirow{4}{*}{ Contribution to NPK removal by crop } & Ideal & $0-10 \%$ \\
\hline & Acceptable & $10-30 \%$ \\
\hline & Tolerable & $30-50 \%$ \\
\hline & Unacceptable & $>50 \%$ \\
\hline \multirow{4}{*}{$\begin{array}{c}\text { Microbial contamination } \\
\text { (Excess infections per } 1000 \text { persons per year) }\end{array}$} & Ideal & $<1$ \\
\hline & Acceptable & $1-3$ \\
\hline & Tolerable & $3-10$ \\
\hline & Unacceptable & $>10$ \\
\hline \multicolumn{3}{|c|}{ Soil quality } \\
\hline \multirow{4}{*}{ Soil profile salinity (mS/m) } & Ideal & 0-200 \\
\hline & Acceptable & $200-400$ \\
\hline & Tolerable & $400-800$ \\
\hline & Unacceptable & $>800$ \\
\hline \multirow{4}{*}{ Soil permeability } & Ideal & None \\
\hline & Acceptable & Slight \\
\hline & Tolerable & Moderate \\
\hline & Unacceptable & Severe \\
\hline \multirow{4}{*}{ Oxidizable carbon loading (kg/ha per month) } & Ideal & $0-400$ \\
\hline & Acceptable & $400-1000$ \\
\hline & Tolerable & $1000-1600$ \\
\hline & Unacceptable & $>1600$ \\
\hline \multirow{4}{*}{$\begin{array}{l}\text { Trace element accumulation } \\
\text { (No of years to reach soil accumulation threshold) }\end{array}$} & Ideal & $>200$ \\
\hline & Acceptable & $150-200$ \\
\hline & Tolerable & $100-150$ \\
\hline & Unacceptable & $<100$ \\
\hline
\end{tabular}


Table 3. Cont.

\begin{tabular}{ccc}
\hline & Fitness for Use & Range \\
\hline Irrigation equipment & & \\
\hline & Ideal & 0 to -0.5 \\
Corrosion of irrigation equipment (Langelier Index) & Acceptable & -0.5 to -1.0 \\
& Tolerable & -1.0 to -2.0 \\
& Unacceptable & $<-2.0$ \\
\hline \multirow{2}{*}{ Scaling (Langelier Index) } & Ideal & 0 to +0.5 \\
& Acceptable & +0.5 to +1.0 \\
& Tolerable & +1.0 to +2.0 \\
& Unacceptable & $>+2.0$ \\
\hline
\end{tabular}

\section{Results}

\subsection{The AF Effluent Fitness for Use}

The output for the DSS showing the generic water quality for irrigation fitness (tier 1) is shown in Table 4. Based on the AF effluent data entered, the DSS calculated the Sodium Adsorption Ratio (SAR) and total dissolved solids of the effluent. The model showed a charge balance error of $-5.3 \%$, which was acceptable. The TDS/EC was unacceptable since the value was 4.46 .

Table 4. The DEWATS effluent showing fitness for agricultural use as determined by the Decision Support System.

\begin{tabular}{|c|c|c|c|c|}
\hline Constituent & Parameter & Unit & Value & \\
\hline \multirow{13}{*}{$\begin{array}{c}\text { Major } \\
\text { constituents }\end{array}$} & Calcium & $\mathrm{mg} \mathrm{L}^{-1}$ & 25 & \\
\hline & Magnesium & $\mathrm{mg} \mathrm{L}^{-1}$ & 20 & \\
\hline & Sodium & $\mathrm{mg} \mathrm{L}^{-1}$ & 55 & \\
\hline & $\mathrm{pH}$ & - & 7.5 & \\
\hline & Electrical conductivity & $\mathrm{mS} \mathrm{m} \mathrm{m}^{-1}$ & 94 & \\
\hline & SAR & $\left(\mathrm{mol} \mathrm{L}^{-1}\right)^{0.5}$ & 2 & \\
\hline & Bicarbonate & $\mathrm{mg} \mathrm{L}^{-1}$ & 231 & \\
\hline & Chloride & $\mathrm{mg} \mathrm{L}^{-1}$ & 49 & \\
\hline & Sulphate & $\mathrm{mg} \mathrm{L}^{-1}$ & 39 & \\
\hline & Total dissolved solids (TDS) & $\mathrm{mg} \mathrm{L}^{-1}$ & 419 & \\
\hline & Suspended solids (SS) & $\mathrm{mg} \mathrm{L}^{-1}$ & 59 & \\
\hline & Charge balance error & - & $-5.30 \%$ * & \\
\hline & $\mathrm{TDS} / \mathrm{EC}$ & - & $4.46^{\#}$ & \\
\hline \multirow{2}{*}{$\begin{array}{l}\text { Biological } \\
\text { constituents }\end{array}$} & E. coli & counts $/ 100 \mathrm{~mL}$ & $4.00 \times 10^{4}$ & \\
\hline & Chemical oxygen demand & $\mathrm{mg} \mathrm{L}^{-1}$ & 303 & \\
\hline \multirow{4}{*}{ Nutrients } & Total inorganic nitrogen $(\mathrm{N})$ & $\mathrm{mg} \mathrm{L}^{-1}$ & 60 & \\
\hline & Total inorganic phosphorus $(\mathrm{P})$ & $\mathrm{mg} \mathrm{L}^{-1}$ & 9 & \\
\hline & Total inorganic potassium (K) & $\mathrm{mg} \mathrm{L}^{-1}$ & 16 & \\
\hline & Trace Element & & $\begin{array}{l}\text { Water } \\
(\mathrm{mg} / \mathrm{L})\end{array}$ & Soil (mg/kg) \\
\hline \multirow{19}{*}{ Trace elements } & Aluminium & & 0 & 0 \\
\hline & Arsenic & & 0 & 0 \\
\hline & Beryllium & & 0 & 0 \\
\hline & Boron & & 0 & 0 \\
\hline & Cadmium & & 0 & 0 \\
\hline & Chromium & & 0 & 0 \\
\hline & Cobalt & & 0 & 0 \\
\hline & Fluoride & & 0 & 0 \\
\hline & Iron & & 0 & 0 \\
\hline & Lead & & 0 & 0 \\
\hline & Lithium & & 0 & 0 \\
\hline & Manganese & & 0 & 0 \\
\hline & Mercury & & 0 & 0 \\
\hline & Molybdenum & & 0 & 0 \\
\hline & Nickel & & 0 & 0 \\
\hline & Selenium & & 0 & 0 \\
\hline & Uranium & & 0 & 0 \\
\hline & Vanadium & & 0 & 0 \\
\hline & Zinc & & 0 & 0 \\
\hline
\end{tabular}

* Ideal, " Unacceptable. 


\subsection{Effects on Soil Quality}

The effects of AF effluent on soil quality were simulated and reported in Table 5. There were no potential effects of AF effluent on soil profile salinity, permeability and oxidizable carbon. The AF effluent was within at least tolerable category for $>50 \%$ of the time. The root zone effects of EC were within the ideal category $100 \%$ of the time. The soil hydraulic conductivity showed some variations, being unacceptable at least $20 \%$ of the time in climatic region 1; clay soil (overhead irrigation; $23 \%$ and surface irrigation; $25 \%$ ), coarse sand soil (surface irrigation; $21 \%$ ) and sandy loam soil; $23 \%$ for all irrigation systems. The effects on soil infiltrability and oxidizable $\mathrm{C}$ were at least within the acceptable category. The exception was oxidizable $C$ loading under surface irrigation, sandy loam soil and within climatic region 3 in which $15 \%$ of the time was unacceptable.

Table 5. The fitness for use of AF effluent with respect to soil quality of various soil types (clay; C, coarse sand; CS and sandy loam; SL), irrigation systems (overhead and surface) in four climatic regions (climatic region 1; CR1, 2; CR2, 3; CR3 and 4; CR4).

\begin{tabular}{|c|c|c|c|c|c|c|c|c|c|c|c|c|c|c|c|c|c|c|}
\hline \multirow{3}{*}{$\begin{array}{c}\text { Irrigation } \\
\text { System }\end{array}$} & \multirow{3}{*}{$\begin{array}{l}\text { Soil } \\
\text { Type }\end{array}$} & \multirow{3}{*}{$\begin{array}{l}\text { Climatic } \\
\text { Region }\end{array}$} & \multirow{2}{*}{\multicolumn{4}{|c|}{$\begin{array}{l}\text { Soil Profile } \\
\text { Salinity } \\
\text { (EC) }\end{array}$}} & \multicolumn{8}{|c|}{ Soil Permeability } & \multicolumn{4}{|c|}{$\begin{array}{c}\text { Soil } \\
\text { Oxidizable C } \\
\text { Loading }\end{array}$} \\
\hline & & & & & & & \multicolumn{4}{|c|}{$\begin{array}{l}\text { Soil Hydraulic } \\
\text { Conductivity }\end{array}$} & \multicolumn{4}{|c|}{$\begin{array}{c}\text { Soil } \\
\text { Infiltrability }\end{array}$} & \multirow[b]{2}{*}{$\mathbf{a}$} & \multirow[b]{2}{*}{$\mathbf{b}$} & \multirow[b]{2}{*}{ c } & \multirow[b]{2}{*}{ d } \\
\hline & & & a & b & c & d & $\mathbf{a}$ & $\mathbf{b}$ & c & d & $\mathbf{a}$ & $\mathbf{b}$ & c & d & & & & \\
\hline \multirow{12}{*}{ Overhead } & \multirow{4}{*}{ C } & CR 1 & 100 & 0 & 0 & 0 & 49 & 15 & 14 & 23 & 82 & 19 & 0 & 0 & 73 & 27 & 0 & 0 \\
\hline & & CR 2 & 100 & 0 & 0 & 0 & 75 & 9 & 7 & 9 & 89 & 12 & 0 & 0 & 55 & 46 & 0 & 0 \\
\hline & & CR 3 & 100 & 0 & 0 & 0 & 91 & 5 & 3 & 2 & 95 & 5 & 0 & 0 & 34 & 66 & 0 & 0 \\
\hline & & CR 4 & 100 & 0 & 0 & 0 & 74 & 12 & 8 & 6 & 83 & 17 & 0 & 0 & 65 & 35 & 0 & 0 \\
\hline & \multirow{4}{*}{ CS } & CR 1 & 100 & 0 & 0 & 0 & 58 & 10 & 13 & 19 & 85 & 15 & 0 & 0 & 80 & 21 & 0 & 0 \\
\hline & & CR 2 & 100 & 0 & 0 & 0 & 75 & 7 & 8 & 10 & 91 & 9 & 0 & 0 & 68 & 33 & 0 & 0 \\
\hline & & CR 3 & 100 & 0 & 0 & 0 & 84 & 6 & 5 & 6 & 95 & 6 & 0 & 0 & 53 & 48 & 0 & 0 \\
\hline & & CR 4 & 100 & 0 & 0 & 0 & 69 & 10 & 10 & 12 & 82 & 18 & 0 & 0 & 67 & 33 & 0 & 0 \\
\hline & \multirow{4}{*}{ SL } & CR 1 & 100 & 0 & 0 & 0 & 51 & 12 & 12 & 25 & 83 & 17 & 0 & 0 & 80 & 20 & 0 & 0 \\
\hline & & CR 2 & 100 & 0 & 0 & 0 & 75 & 8 & 7 & 10 & 90 & 10 & 0 & 0 & 61 & 39 & 0 & 0 \\
\hline & & CR 3 & 100 & 0 & 0 & 0 & 90 & 5 & 3 & 3 & 95 & 5 & 0 & 0 & 42 & 58 & 0 & 0 \\
\hline & & CR 4 & 100 & 0 & 0 & 0 & 68 & 11 & 10 & 12 & 85 & 15 & 0 & 0 & 73 & 27 & 0 & 0 \\
\hline \multirow{12}{*}{ Surface } & \multirow{4}{*}{ C } & CR 1 & 100 & 0 & 0 & 0 & 49 & 15 & 15 & 25 & 58 & 42 & 0 & 0 & 73 & 27 & 0 & 0 \\
\hline & & CR 2 & 100 & 0 & 0 & 0 & 75 & 9 & 6 & 6 & 58 & 42 & 0 & 0 & 53 & 47 & 0 & 0 \\
\hline & & CR 3 & 100 & 0 & 0 & 0 & 91 & 5 & 3 & 2 & 67 & 33 & 0 & 0 & 34 & 66 & 0 & 0 \\
\hline & & CR 4 & 100 & 0 & 0 & 0 & 74 & 12 & 8 & 7 & 52 & 48 & 0 & 0 & 65 & 35 & 0 & 0 \\
\hline & \multirow{4}{*}{ CS } & CR 1 & 100 & 0 & 0 & 0 & 58 & 10 & 13 & 21 & 59 & 41 & 0 & 0 & 81 & 19 & 0 & 0 \\
\hline & & CR 2 & 100 & 0 & 0 & 0 & 75 & 7 & 8 & 8 & 58 & 42 & 0 & 0 & 65 & 35 & 0 & 0 \\
\hline & & CR 3 & 100 & 0 & 0 & 0 & 84 & 5 & 4 & 3 & 67 & 33 & 0 & 0 & 50 & 51 & 0 & 0 \\
\hline & & CR 4 & 100 & 0 & 0 & 0 & 69 & 11 & 10 & 12 & 44 & 56 & 0 & 0 & 75 & 25 & 0 & 0 \\
\hline & \multirow{4}{*}{ SL } & CR 1 & 100 & 0 & 0 & 0 & 51 & 10 & 11 & 23 & 66 & 34 & 0 & 0 & 84 & 15 & 0 & 0 \\
\hline & & CR 2 & 100 & 0 & 0 & 0 & 75 & 8 & 7 & 8 & 58 & 37 & 0 & 0 & 61 & 39 & 0 & 0 \\
\hline & & CR 3 & 100 & 0 & 0 & 0 & 90 & 5 & 3 & 3 & 67 & 34 & 0 & 0 & 35 & 49 & 1 & 15 \\
\hline & & CR 4 & 100 & 0 & 0 & 0 & 68 & 12 & 10 & 9 & 44 & 56 & 0 & 0 & 74 & 26 & 0 & 0 \\
\hline
\end{tabular}

CR; Climatic region, category a; Ideal, b; Acceptable, c; Tolerable, d; Unacceptable, EC; electrical conductivity.

\subsection{Crop Yield and Quality Fitness for Use}

The AF effluent fitness for use was assessed with regards to root zone effects on crop yield due to root zone salinity, $\mathrm{Cl}^{-}, \mathrm{B}$ and $\mathrm{Na}^{+}$, and leaf scorching when wetted (degree of leaf scorching under sprinkler irrigation caused by $\mathrm{Cl}^{-}$and $\left.\mathrm{Na}^{+}\right)$. The results are reported in Table 6A (maize), Table 6B (lettuce) and Table 6C (cabbage).

The AF effluent fitness for use with regards to maize root zone $\mathrm{Cl}^{-}, \mathrm{B}$, and leaf scorching due to $\mathrm{Cl}^{-}$ad $\mathrm{Na}^{+}$was at least within the acceptable category for $>50 \%$ of the time. The root zone EC challenges were reported for clay soil in climatic region 3 and under overhead irrigation, whereby $>50 \%$ of the time for fitness for use fell within the unacceptable category. The same applied to $\mathrm{Na}^{+}$, which was within the unacceptable category for $>50 \%$ of the time except in clay and sandy loam soils within climatic region 3 regardless of irrigation system. 
Table 6. Assessment of AF effluent for fitness-for-Use; impacts of irrigation system and soil type on maize (A), lettuce (B) and cabbage crop (C) yield and quality in different climatic regions $(n=3)$.

\begin{tabular}{|c|c|c|c|c|c|c|c|c|c|c|c|c|c|c|c|}
\hline & & & & & & & & & & & & & & & Mai \\
\hline & & & & & & & & & op & Root & Zone & Eff & fects & & \\
\hline $\begin{array}{l}\text { Irrigation } \\
\text { System }\end{array}$ & $\begin{array}{l}\text { Soil } \\
\text { Type }\end{array}$ & $\begin{array}{c}\text { Climatic } \\
\text { Region }\end{array}$ & & $\mathrm{Cl}^{-}$ & & & & B & & & & E & C & & \\
\hline & & & a & b & c & d & a & b & c & d & a & b & c & d & a \\
\hline & & CR 1 & 100 & 0 & 0 & 0 & 100 & 0 & 0 & 0 & 99 & 1 & 0 & 0 & 95 \\
\hline & & CR 2 & 100 & 0 & 0 & 0 & 100 & 0 & 0 & 0 & 86 & 7 & 4 & 3 & 77 \\
\hline & C & CR 3 & 69 & 11 & 7 & 14 & 100 & 0 & 0 & 0 & 33 & 6 & 7 & 55 & 27 \\
\hline & & CR 4 & 100 & 0 & 0 & & 100 & 0 & 0 & 0 & 91 & 7 & & & \\
\hline & & CR 1 & 100 & 0 & 0 & 0 & 100 & 0 & 0 & 0 & 100 & 0 & 0 & 0 & 99 \\
\hline Overhead & & CR 2 & 100 & 0 & 0 & 0 & 100 & 0 & 0 & 0 & 91 & 5 & 2 & 3 & 88 \\
\hline & CS & CR 3 & 95 & 3 & 2 & 1 & 100 & 0 & 0 & 0 & 81 & 7 & 3 & 9 & 66 \\
\hline & & CR 4 & 100 & 0 & 0 & 0 & 100 & 0 & 0 & 0 & 99 & 1 & 0 & 0 & 94 \\
\hline & & CR 1 & 100 & 0 & 0 & 0 & 100 & 0 & 0 & 0 & 100 & 0 & 0 & 0 & 95 \\
\hline & SL & CR 2 & 100 & 0 & 0 & $\begin{array}{l}0 \\
9\end{array}$ & 100 & 0 & $\begin{array}{l}0 \\
0\end{array}$ & $\begin{array}{l}0 \\
0\end{array}$ & 81 & 11 & 4 & 5 & 68 \\
\hline & & CR 4 & $\begin{array}{l}76 \\
100\end{array}$ & $\begin{array}{l}10 \\
0\end{array}$ & $\begin{array}{l}5 \\
0\end{array}$ & $\begin{array}{l}9 \\
0\end{array}$ & $\begin{array}{l}100 \\
100\end{array}$ & $\begin{array}{l}0 \\
0\end{array}$ & $\begin{array}{l}0 \\
0\end{array}$ & $\begin{array}{l}0 \\
0\end{array}$ & $\begin{array}{l}34 \\
94\end{array}$ & $\begin{array}{l}14 \\
6\end{array}$ & $\begin{array}{l}9 \\
0\end{array}$ & $\begin{array}{l}43 \\
0\end{array}$ & $\begin{array}{l}21 \\
75\end{array}$ \\
\hline & & CR 1 & 100 & 0 & 0 & 0 & 100 & 0 & 0 & 0 & 89 & 6 & 2 & 3 & 84 \\
\hline & & CR 2 & 99 & 1 & 0 & 0 & 100 & 0 & 0 & 0 & 71 & 15 & 8 & 6 & 58 \\
\hline & C & CR 3 & 76 & 11 & 5 & 9 & 100 & 0 & 0 & 0 & 45 & 13 & 6 & 36 & 20 \\
\hline & & CR 4 & 100 & 0 & 0 & 0 & 100 & 0 & 0 & 0 & 95 & 5 & 0 & 0 & 83 \\
\hline & & CR 1 & 100 & 0 & & 0 & 100 & 0 & 0 & 0 & 90 & 5 & 2 & 3 & 89 \\
\hline Surface & & CR 2 & 100 & 0 & 0 & 0 & 100 & 0 & 0 & 0 & 99 & 1 & 1 & 0 & 96 \\
\hline & Cs & CR 3 & 99 & 1 & 0 & 0 & 100 & 0 & 0 & 0 & 92 & 8 & 0 & 0 & 73 \\
\hline & & CR 4 & 100 & 0 & 0 & 0 & 100 & 0 & 0 & 0 & 97 & 3 & 0 & 0 & 88 \\
\hline & & CR 1 & 100 & 0 & 0 & 0 & 100 & 0 & 0 & 0 & 90 & 5 & 2 & 3 & 85 \\
\hline & & CR 2 & 100 & 0 & 0 & 0 & 100 & 0 & 0 & 0 & 76 & 14 & 5 & 5 & 62 \\
\hline & SL & CR 3 & 79 & 9 & 4 & 9 & 100 & 0 & 0 & 0 & 62 & 12 & 5 & 22 & 26 \\
\hline & & CR 4 & 100 & 0 & 0 & 0 & 100 & 0 & 0 & 0 & 95 & 5 & 0 & 0 & 79 \\
\hline & & & & & & & & & & & & & & & Lett \\
\hline & & & & & & & ant $R$ & oot & Zon & Effe & & & & & \\
\hline $\begin{array}{l}\text { Irrigation } \\
\text { System }\end{array}$ & Soil & Climatic & & $\mathrm{Cl}^{-}$ & & & & B & & & & E & C & & \\
\hline & & & a & b & c & d & a & b & c & d & $\mathbf{a}$ & b & c & d & \\
\hline & & CR 1 & 100 & 0 & 0 & 0 & 100 & 0 & 0 & 0 & 97 & 3 & 0 & 0 & \\
\hline & C & CR 2 & 100 & 0 & 0 & 0 & 100 & 0 & 0 & 0 & 98 & 2 & 0 & 0 & \\
\hline & & CR 3 & 100 & 0 & 0 & 0 & 100 & 0 & 0 & 0 & 96 & 4 & 0 & 0 & \\
\hline & & CR 4 & 100 & 0 & 0 & 0 & 100 & 0 & 0 & 0 & 90 & 8 & 2 & 0 & \\
\hline & & CR 1 & 100 & 0 & 0 & 0 & 100 & 0 & 0 & 0 & 98 & 2 & 0 & 0 & \\
\hline Overhead & & CR 2 & 100 & 0 & 0 & 0 & 100 & 0 & 0 & 0 & 82 & 9 & 5 & 4 & \\
\hline & CS & CR 3 & 88 & 4 & 4 & 4 & 100 & 0 & 0 & 0 & 56 & 13 & 2 & 29 & \\
\hline & & CR 4 & 97 & 2 & 1 & 0 & 100 & 0 & 0 & 0 & 63 & 14 & 8 & 16 & \\
\hline & & CR 1 & 87 & 5 & 1 & 7 & 100 & 0 & 0 & 0 & 38 & 14 & 11 & 38 & \\
\hline & SL & $\mathrm{CR} 2$ & 100 & 0 & 0 & 0 & 100 & 0 & 0 & 0 & 98 & 2 & 0 & 0 & \\
\hline & $\mathrm{SL}$ & CR 3 & 100 & 0 & 0 & 0 & 100 & 0 & 0 & 0 & 98 & 1 & 1 & 0 & \\
\hline & & CR 4 & 100 & 0 & 0 & 0 & 100 & 0 & 0 & 0 & 92 & 7 & 1 & 0 & \\
\hline & & CR 1 & 100 & 0 & 0 & 0 & 100 & 0 & 0 & 0 & 78 & 13 & 6 & 3 & \\
\hline & & CR 2 & 100 & 0 & 0 & 0 & 100 & 0 & 0 & 0 & 81 & 10 & 6 & 3 & \\
\hline & C & CR 3 & 100 & 0 & 0 & 0 & 100 & 0 & 0 & 0 & 79 & 12 & 6 & 3 & \\
\hline & & CR 4 & 100 & 0 & 0 & 0 & 100 & 0 & 0 & 0 & 75 & 15 & 7 & 3 & \\
\hline & & CR 1 & 100 & 0 & 0 & 0 & 100 & 0 & 0 & 0 & 95 & 5 & 1 & 0 & \\
\hline Surface & & CR 2 & 100 & 0 & 0 & 0 & 100 & 0 & 0 & 0 & 81 & 10 & 6 & 3 & \\
\hline & CS & CR 3 & 86 & 6 & 1 & 7 & 100 & 0 & 0 & 0 & 41 & 11 & 9 & 39 & \\
\hline & & CR 4 & 100 & 0 & 0 & 0 & 100 & 0 & 0 & 0 & 94 & 6 & 0 & 0 & \\
\hline & & CR 1 & 88 & 4 & 4 & 4 & 100 & 0 & 0 & 0 & 63 & 7 & 1 & 29 & \\
\hline & & CR 2 & 100 & 0 & 0 & 0 & 100 & 0 & 0 & 0 & 99 & 1 & 0 & 0 & \\
\hline & SL & CR 3 & 100 & 0 & 0 & 0 & 100 & 0 & 0 & 0 & 99 & 1 & 0 & 0 & \\
\hline & & CR 4 & 100 & 0 & 0 & 0 & 100 & 0 & 0 & 0 & 96 & 3 & 1 & 0 & \\
\hline & & & & & & & & & & & & & & (C) & Cabb \\
\hline & & & & & & & & & ant & Root & Zone & e Eff & fects & & \\
\hline $\begin{array}{l}\text { Irrigation } \\
\text { System }\end{array}$ & $\begin{array}{l}\text { Soil } \\
\text { Type }\end{array}$ & $\begin{array}{c}\text { Climatic } \\
\text { Region }\end{array}$ & & $\mathrm{Cl}^{-}$ & & & & B & & & & E & C & & \\
\hline & & & a & $\mathbf{b}$ & c & $d$ & a & b & c & d & a & b & c & d & a \\
\hline & & CR 1 & 100 & 0 & 0 & 0 & 100 & 0 & 0 & 0 & 85 & 12 & 3 & 0 & 100 \\
\hline & & CR 2 & 100 & 0 & 0 & 0 & 100 & 0 & 0 & 0 & 87 & 12 & 1 & 0 & \\
\hline & C & CR 3 & 100 & 0 & 0 & 0 & 100 & 0 & 0 & 0 & 88 & 8 & 4 & 1 & 100 \\
\hline & & CR 4 & 100 & 0 & 0 & 0 & 100 & 0 & 0 & 0 & 75 & 16 & 12 & 13 & 100 \\
\hline & & CR 1 & & & 0 & & 100 & 0 & 0 & & 42 & & & & \\
\hline Overhead & & CR 2 & 100 & 0 & 0 & 0 & 100 & 0 & 0 & 0 & 54 & 20 & 12 & 14 & 100 \\
\hline & CS & CR 3 & 88 & 4 & 4 & 4 & 100 & 0 & 0 & 0 & 4 & 4 & 7 & 84 & 100 \\
\hline & & CR 4 & 97 & 2 & 1 & 0 & 100 & 0 & 0 & 0 & 35 & 58 & 7 & 0 & 100 \\
\hline & & CR 1 & 87 & 5 & 1 & 7 & 100 & 0 & 0 & 0 & 18 & 13 & 16 & 54 & 100 \\
\hline & & & & 0 & 0 & 0 & 100 & 0 & 0 & 0 & 87 & 11 & 1 & 0 & \\
\hline & SL & CR 3 & 100 & 0 & 0 & 0 & 100 & 0 & 0 & 0 & 92 & 5 & 3 & 0 & 100 \\
\hline & & CR 4 & 100 & 0 & 0 & 0 & 100 & 0 & 0 & 0 & 83 & 13 & 3 & 0 & 97 \\
\hline & & & & & 0 & & & 0 & 0 & & & & & 0 & \\
\hline & & CR 2 & 100 & 0 & 0 & 0 & 100 & 0 & 0 & 0 & 85 & 15 & 0 & 0 & 100 \\
\hline & C & CR 3 & 100 & 0 & 0 & 0 & 100 & 0 & 0 & 0 & 88 & 8 & 4 & 1 & 100 \\
\hline & & CR 4 & 100 & 0 & 0 & 0 & 100 & 0 & 0 & 0 & 44 & 24 & 14 & 18 & 100 \\
\hline & & CR 1 & 100 & 0 & 0 & 0 & 100 & 0 & 0 & 0 & 44 & 53 & 4 & 0 & 100 \\
\hline Surface & & & & 0 & 0 & 0 & 100 & 0 & 0 & 0 & 43 & 26 & 13 & 11 & \\
\hline & CS & CR 3 & 86 & 6 & 1 & 7 & 100 & 0 & 0 & 0 & 18 & 9 & 15 & 58 & 100 \\
\hline & & CR 4 & 100 & 0 & 0 & 0 & 100 & 0 & 0 & 0 & 36 & 58 & 7 & 0 & 100 \\
\hline & & & & & 4 & & & 0 & 0 & & 18 & & 16 & 54 & \\
\hline & & CR 2 & 100 & 0 & 0 & 0 & 100 & 0 & 0 & 0 & 82 & 17 & 1 & 0 & 100 \\
\hline & SL & & 100 & 0 & 0 & 0 & 100 & 0 & 0 & 0 & 97 & 3 & 0 & 0 & 100 \\
\hline & & CR 4 & 100 & 0 & 0 & 0 & 100 & 0 & 0 & 0 & 91 & 9 & 1 & 0 & 100 \\
\hline
\end{tabular}


There are no parameters for plant root zone effects of $\mathrm{Na}^{+}$in lettuce, however, the plant root zone $\mathrm{Cl}^{-}$and $\mathrm{B}$, and leaf scorching due to $\mathrm{Cl}^{-}$and $\mathrm{Na}^{+}$were reported. These were at least acceptable for $>50 \%$ of the time. The plant root zone EC effects were unacceptable for $>25 \%$ of the time in climatic regions 1 and 3 (coarse sand soil and sandy loam soil), regardless of irrigation system, however, the values were below $50 \%$ of the time.

The cabbage root zone effects due to $\mathrm{Cl}^{-}, \mathrm{Na}^{+}, \mathrm{B}$ and leaf scorching effects of $\mathrm{Cl}^{-}$and $\mathrm{Na}^{+}$were within at least acceptable category for $>50 \%$ of the time. The effects of root zone EC were unacceptable ( $>50 \%$ of the time) in climatic region 1 (sandy loam soil) and climatic region 3 (coarse sandy soil), regardless of irrigation system.

\subsection{Contribution to $N$ and $P$ Removal}

The K loading only significantly differed $(p<0.05)$ amongst four climatic regions (Table A1 in Appendix A). Higher loading was reported for climatic region 3 followed by 2, 4 and 1 in that chronological order (Figure 2).

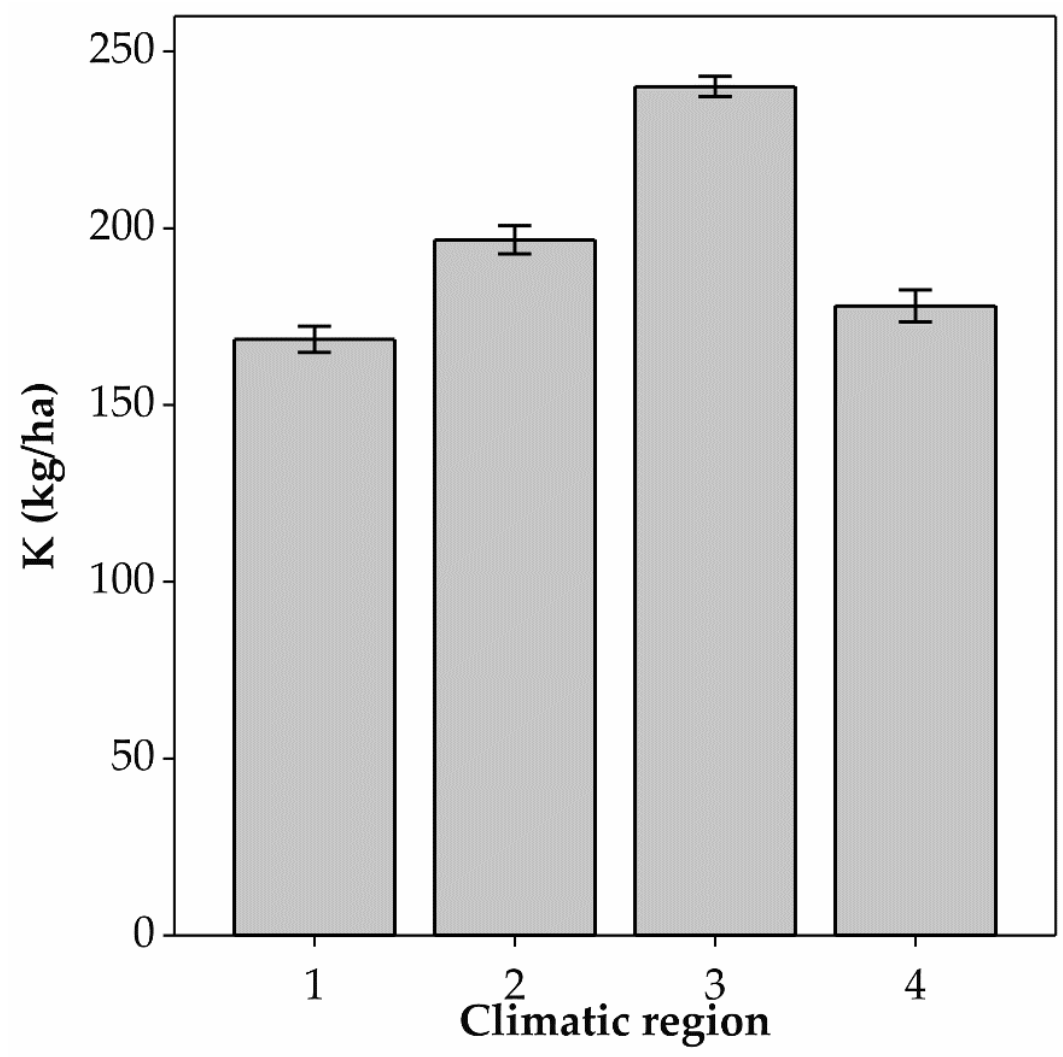

Figure 2. Mean values $(n=144)$ for $\mathrm{K}$ loading in two cropping systems of the four different climatic regions (1; Climatic region 1, 2; Climatic region 2, 3; Climatic region 3 and 4; Climatic region 4) of South Africa.

There were no parameters for the contribution of AF effluent to N, $\mathrm{P}$ and $\mathrm{K}$ removal by lettuce, hence $\mathrm{N}$ and $\mathrm{P}$ were reported for maize and cabbage in Figures 3 and 4, respectively.

The predicted contribution of AF effluent to maize $\mathrm{N}$ and $\mathrm{P}$ removal was unacceptable for $>50 \%$ of the time regardless of soil type, climatic region and irrigation system.

The predicted cabbage $\mathrm{N}$ and $\mathrm{P}$ uptake showed different patterns, whereby $\mathrm{N}$ uptake was at least tolerable for $>50 \%$ of the time in all irrigation systems within climatic region 4 under clay and sandy loam soil types. Phosphorus uptake was at least tolerable for $>50 \%$ of the time in climatic region 4 regardless of soil type and irrigation system. 

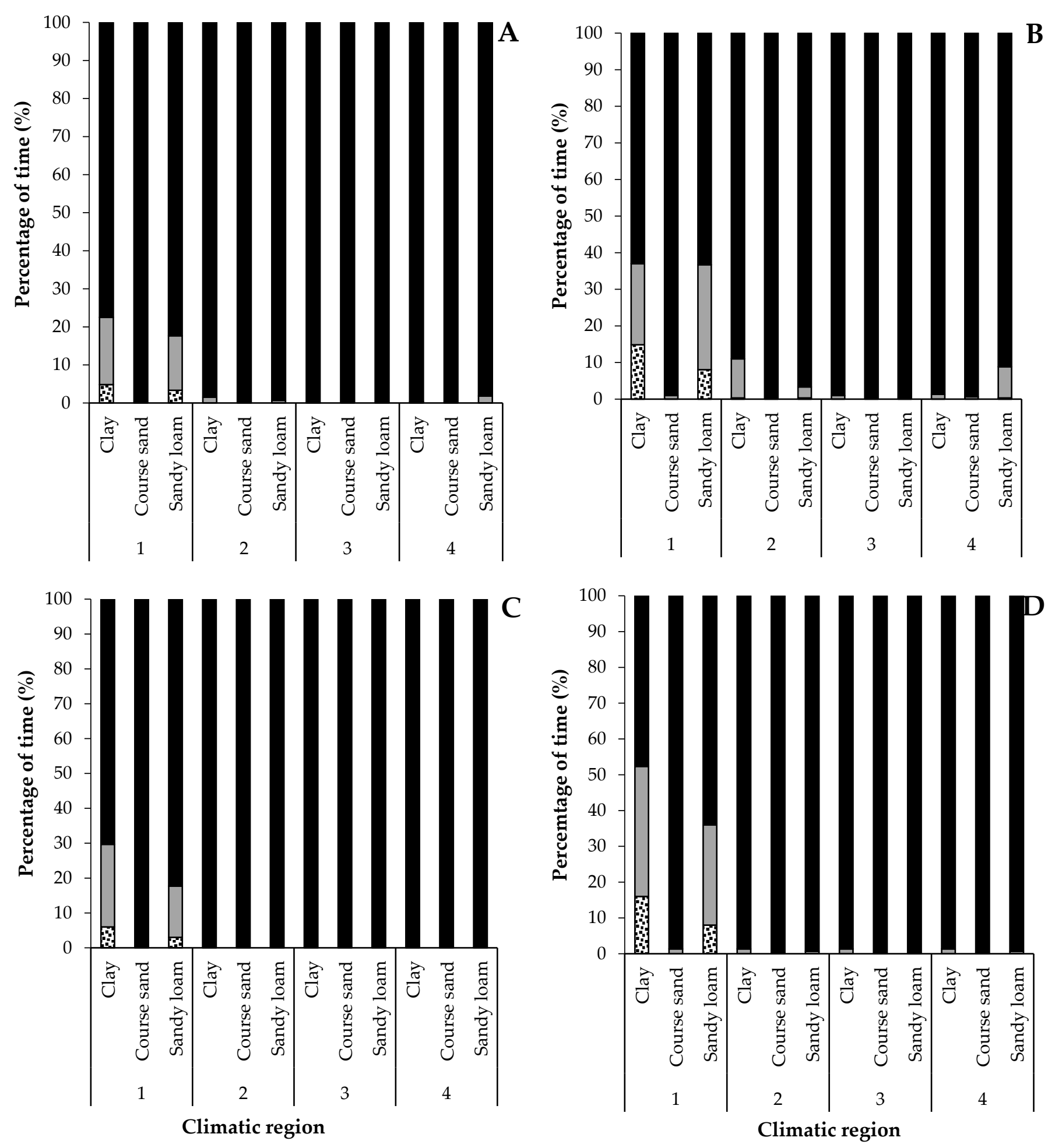

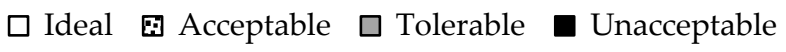

$\square$ Ideal $\square$ Acceptable $\square$ Tolerable $\square$ Unacceptable

Figure 3. Assessment of AF effluent for fitness-for-use; impacts of irrigation system and soil type on maize crop yield and quality in different climatic regions $(n=3)$. (A) (Maize, N uptake, overhead irrigation), (B) (Maize, P uptake, overhead irrigation), (C) (Maize, $\mathrm{N}$ uptake, surface irrigation), (D) (Maize, $\mathrm{N}$ uptake, surface irrigation). 1; Climatic region 1, 2; Climatic region 2, 3; Climatic region 3 and 4; Climatic region 4. 

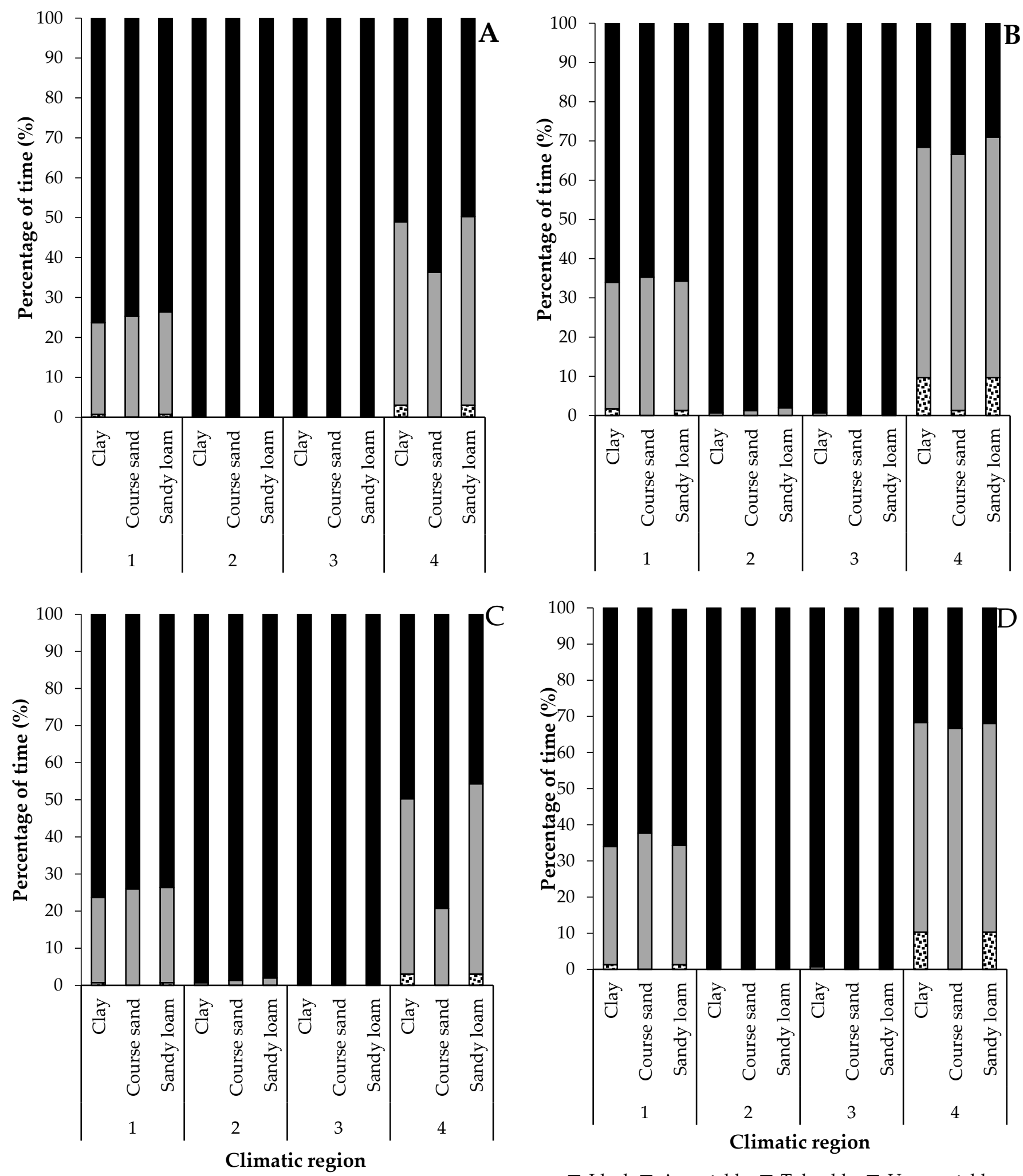

$\square$ Ideal Acceptable $\square$ Tolerable Unacceptable

$\square$ Ideal $\mathbf{t}$ Acceptable $\square$ Tolerable $\square$ Unacceptable

Figure 4. Assessment of AF effluent for fitness-for-Use; impacts of irrigation system and soil type on cabbage crop yield and quality in different climatic regions $(n=3)$. (A) (Cabbage, $\mathrm{N}$ uptake, overhead irrigation), (B) (Cabbage, $\mathrm{P}$ uptake, overhead irrigation), (C) (Cabbage, $\mathrm{N}$ uptake, surface irrigation), (D) (Cabbage, $\mathrm{N}$ uptake, surface irrigation). 1; Climatic region 1, 2; Climatic region 2, 3; Climatic region 3 and 4; Climatic region 4. 
The predicted total nutrients applied in two cropping systems (maize vs. cabbage and maize vs. lettuce rotations) are reported in Figure 5. A significant difference in N and $\mathrm{P}$ applied $(p<0.05)$ was reported for various climatic regions and cropping systems (Table A1). The $\mathrm{N}$ loading was generally higher in climatic region 3 . The least values were predicted in maize and lettuce rotation from climatic regions 1 and 4 . The P loading followed a different pattern, characterized by low values (maize and lettuce rotation) in climatic regions 1, 2 and 4, while in climatic region 3 the values were comparable between the two cropping systems.
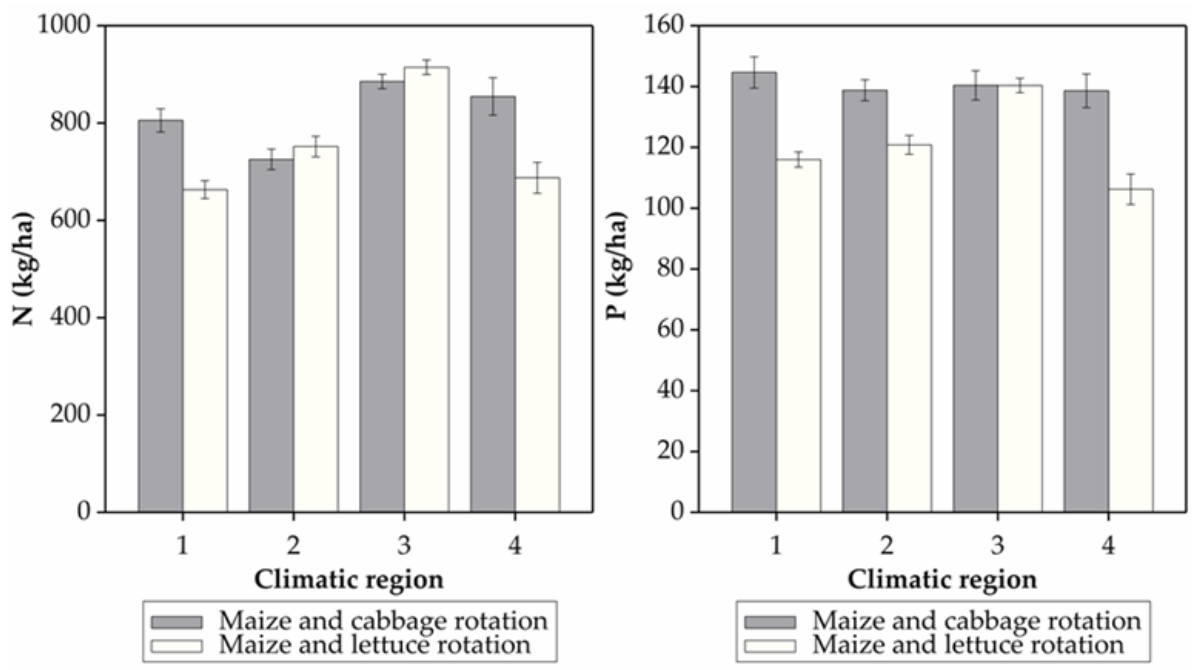

Figure 5. Simulated nitrogen $(\mathrm{N})$, phosphorus $(\mathrm{P})$ and potassium $(\mathrm{K})$ (mean \pm standard error of mean deviation; $n=72$ ) applied through irrigation using DEWATS effluent to three crops on four different soil types. 1; Climatic region 1, 2; Climatic region 2, 3; Climatic region 3 and 4; Climatic region 4.

\subsection{Trace Elements}

The AF effluent is very ideal for agricultural use, and it was predicted that no trace elements are expected to accumulate for $>200$ years of irrigation (Figure 6).

\begin{tabular}{|c|c|c|c|c|c|}
\hline $\begin{array}{l}\text { Fitness for } \\
\text { use category }\end{array}$ & \multicolumn{5}{|c|}{$\begin{array}{l}\text { Average number of years of irrigation with a certain amount of } \\
\text { effluent before trace elements reach accumulation thresholds in }\end{array}$} \\
\hline Ideal & \multicolumn{5}{|c|}{$>200$ years to reach soil accumulated thresholds } \\
\hline Acceptable & \multicolumn{5}{|c|}{150 to 200 years to reach soil accumulated thresholds } \\
\hline Tolerable & \multicolumn{5}{|c|}{100 to 150 years to reach soil accumulated thresholds } \\
\hline Unacceptable & \multicolumn{5}{|c|}{$<100$ years to reach soil accumulated thresholds } \\
\hline Trace element & $\begin{array}{l}\text { Soil } \\
\text { accumulation } \\
\text { threshold } \\
(\mathrm{mg} / \mathrm{kg})\end{array}$ & $\begin{array}{l}\text { No. of years to } \\
\text { reach soil } \\
\text { accumulation } \\
\text { thresholds }\end{array}$ & Trace element & $\begin{array}{l}\text { Soil } \\
\text { accumulation } \\
\text { threshold } \\
(\mathrm{mg} / \mathrm{kg})\end{array}$ & $\begin{array}{l}\text { No. of years } \\
\text { to reach soil } \\
\text { accumulation } \\
\text { thresholds }\end{array}$ \\
\hline $\mathrm{Al}$ & 2500 & Infinite & $\mathrm{Li}$ & 1250 & Infinite \\
\hline As & 50 & Infinite & $\mathrm{Mn}$ & 100 & Infinite \\
\hline Be & 50 & Infinite & $\mathrm{Hg}$ & 1 & Infinite \\
\hline $\mathrm{Cd}$ & 5 & Infinite & Mo & 5 & Infinite \\
\hline $\mathrm{Cr}$ & 50 & Infinite & $\mathrm{Ni}$ & 100 & Infinite \\
\hline Co & 25 & Infinite & $\mathrm{Se}$ & 10 & Infinite \\
\hline $\mathrm{Cu}$ & 100 & Infinite & $\mathrm{U}$ & 5 & Infinite \\
\hline $\mathrm{F}$ & 1000 & Infinite & $\mathrm{V}$ & 50 & Infinite \\
\hline $\mathrm{Fe}$ & 2500 & Infinite & $\mathrm{Zn}$ & 100 & Infinite \\
\hline $\mathrm{Pb}$ & 100 & Infinite & & & \\
\hline
\end{tabular}

Figure 6. Effects of AF effluent irrigation on the accumulation of trace elements. 


\subsection{Irrigation Equipment}

There are no significant effects of AF effluent characteristics (suspended solids, $\mathrm{pH}$, $\mathrm{Mn}, \mathrm{Fe}$ and E. coli) on potential clogging of drippers as all these were within at least the tolerable ranges (Table 7).

Table 7. The fitness for use of AF effluent based on selected characteristics that cause clogging of drippers.

\begin{tabular}{|c|c|c|c|}
\hline & Fitness for Use Category & Range & Observed Value \\
\hline \multirow{4}{*}{$\begin{array}{l}\text { Suspended solids } \\
\qquad\left(\mathrm{mg} \mathrm{L}^{-1}\right)\end{array}$} & Ideal & $<50$ & \multirow{4}{*}{91} \\
\hline & Acceptable & $50-75$ & \\
\hline & Tolerable & $75-100$ & \\
\hline & Unacceptable & $>100$ & \\
\hline \multirow{4}{*}{$\mathrm{pH}$} & Ideal & $<7.0$ & \multirow{4}{*}{7.6} \\
\hline & Acceptable & $7.0-7.5$ & \\
\hline & Tolerable & $7.5-8.0$ & \\
\hline & Unacceptable & $>8$ & \\
\hline \multirow{4}{*}{$\begin{array}{l}\text { Manganese } \\
\left(\mathrm{mg} \mathrm{L}^{-1}\right)\end{array}$} & Ideal & $<0.1$ & \multirow[t]{4}{*}{0.0} \\
\hline & Acceptable & $0.1-0.5$ & \\
\hline & Tolerable & $0.5-1.5$ & \\
\hline & Unacceptable & $>1.5$ & \\
\hline \multirow{4}{*}{$\begin{array}{l}\text { Total Iron } \\
\left(\mathrm{mg} \mathrm{L}^{-1}\right)\end{array}$} & Ideal & $<0.2$ & \multirow[t]{4}{*}{0.0} \\
\hline & Acceptable & $0.2-0.5$ & \\
\hline & Tolerable & $0.5-1.5$ & \\
\hline & Unacceptable & $>1.5$ & \\
\hline \multirow{4}{*}{$\begin{array}{c}\text { E. coli } \\
(106 \text { per } 100 \mathrm{~mL})\end{array}$} & Ideal & $<1$ & \multirow[t]{4}{*}{0.025} \\
\hline & Acceptable & $1-2$ & \\
\hline & Tolerable & $2-5$ & \\
\hline & Unacceptable & $>5$ & \\
\hline
\end{tabular}

Determined by the potential of an irrigation water constituent to cause clogging of drippers.

There were no predicted effects of effluent on the corrosion and scaling of irrigation equipment as determined by the Langelier index (Table 8). The fitness for use was within the ideal category.

Table 8. Fitness for Use Category determined by the corrosion or scaling potential indicated by the Langelier Index.

\begin{tabular}{ccccc}
\hline $\begin{array}{c}\text { Fitness for Use } \\
\text { Category }\end{array}$ & $\begin{array}{c}\text { Corrosion } \\
\text { (Langelier Index) }\end{array}$ & $\begin{array}{c}\text { Observed } \\
\text { Score }\end{array}$ & $\begin{array}{c}\text { Scaling } \\
\text { (Langelier Index) }\end{array}$ & $\begin{array}{c}\text { Observed } \\
\text { Score }\end{array}$ \\
\hline Ideal & 0 to -0.5 & -0.37 & 0 to +0.5 & Not scaling \\
Acceptable & -0.5 to -0.1 & & +0.5 to +0.1 & \\
Tolerable & -0.1 to -2.0 & & +0.1 to +2.0 & \\
Unacceptable & $<-2.0$ & & $>+2.0$ & \\
\hline
\end{tabular}

\subsection{Microbial Contamination}

The predicted impacts of AF effluent on microbial contamination varied with crop type and irrigation system (Table 9). There are no pathogen risks for irrigating maize with AF effluent regardless of the irrigation system used. However, this differs with cabbage and lettuce, which predicted risks of 82.6 and 101.1 excess infections per 1000 people, especially when overhead irrigation is used. 
Table 9. The fitness for use of AF effluent with focus on predicted excess infections per 1000 people per annum depending on crop type and irrigation system.

\begin{tabular}{|c|c|c|c|}
\hline Crop & Irrigation System & Category & $\begin{array}{c}\text { Predicted Excess Infections } \\
\text { per } 1000 \text { People }\end{array}$ \\
\hline \multirow{8}{*}{ Maize } & \multirow{4}{*}{ Overhead } & Ideal & 0 \\
\hline & & Acceptable & \\
\hline & & Tolerable & \\
\hline & & Unacceptable & \\
\hline & \multirow{4}{*}{ Surface } & Ideal & 0 \\
\hline & & Acceptable & \\
\hline & & Tolerable & \\
\hline & & Unacceptable & \\
\hline \multirow{8}{*}{ Cabbage } & \multirow{4}{*}{ Overhead } & Ideal & \\
\hline & & Acceptable & \\
\hline & & Tolerable & \\
\hline & & Unacceptable & 82.6 \\
\hline & \multirow{4}{*}{ Surface } & Ideal & 0 \\
\hline & & Acceptable & \\
\hline & & Tolerable & \\
\hline & & Unacceptable & \\
\hline \multirow{8}{*}{ Lettuce } & \multirow{4}{*}{ Overhead } & Ideal & \\
\hline & & Acceptable & \\
\hline & & Tolerable & \\
\hline & & Unacceptable & 101.1 \\
\hline & \multirow{4}{*}{ Surface } & Ideal & 0 \\
\hline & & Acceptable & \\
\hline & & Tolerable & \\
\hline & & Unacceptable & \\
\hline
\end{tabular}

\section{Discussion}

\subsection{The AF Effluent Fitness for Use}

The charge balance error shows the reliability of analytical results from a specific sample. In principle, the anions should balance out the cations. A charge balance of $5 \%$ is the most ideal, however, the $5.3 \%$ reported during the study indicates that the analysis results were reliable. However, the unacceptable TDS/EC ratio of 4.46, was attributed to the EC value of $94.4 \mathrm{mS} / \mathrm{m}$ instead of $89 \mathrm{~ms} / \mathrm{m}$ required to at least get the tolerable value. This discrepancy could have been attributed to the use of collated AF effluent analysis results, which have been done by different individuals. Although du Plessis et al. [12] suggested that the DSS should be parameterised using water quality from a single analysis, there were no comprehensive AF effluent quality results available from a single analysis. Literature data were thus used to run the simulation, and the difference from the unacceptable to the tolerable TDS/EC was not large (4.46 instead of 4.71), proving that the literature results were credible.

\subsection{Effects on Soil Quality}

The predicted soil root zone salinity was ideal (100\% of the time) for FFU in all climatic regions soil, soil type and irrigation systems (Table 5). This is because the EC of the AF effluent was within the most ideal range of $0-200 \mathrm{mS} \mathrm{m}^{-1}$ for fitness of use which is expected in domestic wastewater $[25,26]$. This implies that AF effluent has no negative impacts on soil salinity.

Hydraulic conductivity is the rate at which water moves through a porous material, in this case, the bulk soil. This is affected by the interaction of the sodium adsorption ratio (SAR) and the EC. There are certain levels to which soil water EC should be reduced to effect a $10-15 \%$ reduction in hydraulic conductivity in a soil with a specific exchangeable 
sodium percentage (ESP) [12]. Generally, as the soil EC increases at a certain soil ESP the risk to the hydraulic conductivity decreases. For irrigation water with a SAR of 2-3 and EC of $>60 \mathrm{mS} \mathrm{m}^{-1}$, the degree of reduced soil permeability is none [12], therefore the expected degree of reduction in hydraulic conductivity due to irrigation with AF effluent was very low (Table 5) because the AF effluent has a SAR of 2 and EC of $94 \mathrm{mS} \mathrm{m}^{-1}$ (Table 4).

In addition, soil infiltrability can be reduced by raindrops or irrigation water action [12]. However, the predicted reduction in soil infiltrability was insignificant regardless of climatic region, soil type and irrigation system (Table 5), implying that overhead irrigation can be used to apply AF effluent with no potential problems.

The simulated oxidisable $C$ loading in DEWATS irrigated soils was very low (Table 5). This was expected since the mean AF effluent chemical oxygen demand (COD) was $303 \mathrm{mg} / \mathrm{L}$ (Table 4), which was much lower than the maximum limit of $5000 \mathrm{mg} \mathrm{L}^{-1}$ for irrigating with $50 \mathrm{~m}^{3}$ of effluent per day [13]. Implying that oxidisable carbon loading is not a challenge when irrigating using AF effluent regardless of climatic region and soil type.

\subsection{Crop Yield and Quality Fitness for Use}

The impacts of AF effluent on root zone salinity and leaf scorching showed to vary across crop types. The root zone EC was unacceptable for $>50 \%$ of the time in climatic region 3 and clay soil (maize). Variations in the effects of EC across climatic regions can be attributed to differences in climatic conditions. Large volumes of effluent can be applied in climatic region 3, a desert and hot arid region (Table 1), characterised by high evapotranspiration (ET) and low rainfall. du Plessis et al. [12] stated that the effects of salinity are not generally caused by volumes of effluent applied but by the amount of water extracted by plants, altering the soil water potential through the salinization process. Therefore, the root zone EC effects on cabbage reported in climatic region 1 (sandy loam soil) and climatic region 3 (coarse sandy soil), regardless of irrigation system means that in such areas the crop is likely to suffer from salinity.

Salinisation decreases cabbage yields as reported in soils irrigated with wastewater under arid conditions $[27,28]$. Therefore, soil salinity in maize and cabbages are grown in climatic region 3 of South Africa can be managed through various methods such as the application of freshwater based on the leaching fraction to remove excess salts from the topsoil as recommended by the Food and Agriculture Organisation guidelines [10].

\subsection{Contribution to N and P Removal}

Most countries in the Sub Saharan region are food insecure [29], hence agricultural use of treated wastewater from onsite systems such as DEWATS should help alleviate this problem by increasing crop yield and quality. However, the AF effluent proved to be unfit for maize production with regards to contribution to $\mathrm{N}$ and $\mathrm{P}$ uptake. This is contrary to reports given by some authors $[30,31]$ that treated wastewater can supply nutrients to increase crop yields, which is not always the case. Overapplication of nutrients such as $\mathrm{N}$ is likely to cause delayed flowering and uneven ripening. Meaning that certain site and crop-specific management practices such as effluent dilution as suggested by Food and Agriculture Organisation [10] should be considered in flowering crops such as maize field irrigated with AF effluent. Therefore, in this case, the AF effluent should be diluted to reduce the concentrations of $\mathrm{N}$ and $\mathrm{P}$ before being applied to maize crops regardless of soil type and climatic region. Alternatively, the effluent can be applied on a larger land area to meet the crop nutrient requirements as recommended by FAO [32].

The contribution of AF effluent to $\mathrm{N}$ and $\mathrm{P}$ uptake in cabbage depended on soil type and climatic region (Figure 3). The $\mathrm{N}$ and $\mathrm{P}$ uptake was tolerable in climatic region 4; clay and sandy loam soils because less amount of effluent should be applied in such areas as they are characterised by high rainfall and low evapotranspiration rates. Therefore, AF effluent should be diluted if it is used to irrigate cabbage in South African climatic regions 
1-3 regardless of soil type. However, in such circumstances, a freshwater supply should be available, and more land will be required.

The fitness of AF effluent for agricultural use as defined by the DSS is the ability to provide nutrients for crop growth, followed by their adequate uptake and minimal loading into the soil. The nutrients loaded in the soil are potential pollutants, especially $\mathrm{N}$ and P. Nitrogen can be leached down the soil profile leading to groundwater contamination [33] and phosphorus can be washed to nearby surface water where it will lead to non-point pollution [22]. Based on the assessment, cabbage grown in climatic region 4 may effectively remove nutrients from the soil (Figure 4), thereby minimising potential environmental pollution.

Apart from providing nutrients for food crop production, the agricultural use of AF effluent may minimise amounts of nutrients discharged into the environment, thereby acting as a sustainable waste management option that can be adopted by municipalities. Therefore, agricultural systems that can effectively remove nutrients while balancing crop quality and minimal pollution are desirable. The results reported in Figure 5 showed that a maize and cabbage rotation system is loads more nutrients than maize and lettuce.

The model predicted high $\mathrm{K}$ loading in climatic region 3 than climatic regions 1 (warm temperate areas) and 4 (cold arid) (Figure 2) due to high evapotranspiration rates and subsequent irrigation requirements. Therefore, low $\mathrm{K}$ soils in climatic region 3 are likely to benefit from AF effluent irrigation.

\subsection{Trace Elements}

Trace elements are hazardous to the environment, crops and end consumers of the products irrigated with wastewater. However, the accumulation of trace elements even when soils are irrigated with AF effluent for over 200 years were negligible (Figure 6). Therefore, AF effluent can be safely used without significantly loading heavy metals into the soil. This corroborates findings reported by Levy et al. [34] that domestic treated wastewater is low in heavy metals unless contaminated with industrial effluent. Furthermore, one advantage of an on-site system such as DEWATS over conventional wastewater treatment systems is that it minimises the chances of having industrial effluent being illegally discharged into the treatment system.

\subsection{Irrigation Equipment}

The AF effluent contained very low concentrations of $\mathrm{Mn}$, Fe and microorganisms and tolerable levels of suspended solids with a $\mathrm{pH}$ that has no significant impacts on clogging of the irrigation equipment (Table 7), scaling and corrosion of irrigation equipment (Table 8). However, studies by Dirwai et al. [35] showed that the AF effluent can clog moisture irrigation technology (MIT) pipes if not filtered or flushed from the system. This implies that even though the AF effluent is within tolerable ranges for fitness for use with regards to clogging of drippers, measures such as acidification and installation of filters with a backwash system may need to be taken into consideration as mitigation strategies. Therefore, AF effluent can be used for directed irrigation using a drip system, which is a highly recommended method by the WHO to minimise microbial risks and increase irrigation efficiency [10] but precautions should be taken to minimise clogging problems.

\subsection{Microbial Contamination}

Human health safety in the agricultural use of treated wastewater is of concern. The use of AF effluent should abide by the World Health Organisation [16] guidelines. Maize crops showed to be at less risk to microbial contamination as reported by Farhadkhani et al. [36]. This is because the cob is produced inside the husk and the crop cannot be consumed uncooked. Therefore, any irrigation system (overhead and surface) may be used for maize production. The cabbage and lettuce showed to be at higher microbial risks since they can be eaten as salads, hence any irrigation system that can wet their leaves is undesirable. It is therefore advisable to use surface irrigation for crops such as cabbage 
and lettuce. Furthermore, if overhead irrigation is to be used, the AF effluent should be further treated to deactivate pathogens to allow unrestricted use as recommended by the World Health Organisation [16]. However, such post-treatments include ozonation and UV radiation but studies by De Sanctis et al. [37] reported that $C$. perfringens may not be completely removed. Therefore, drip or surface irrigation are still cost-effective methods to minimise microbial contamination in high-risk crops such as cabbage and lettuce.

\section{Conclusions}

The AF effluent can be used in any soil type and South African climatic region without negatively affecting soil quality parameters such as root zone salinity, soil infiltrability, hydraulic conductivity and oxidisable carbon loading, regardless of climatic region and irrigation system.

The root zone salinity problems are expected in cabbage and maize crops, especially in the South African climatic region 3. Implying that salinity management practices such as salt leaching should be done in such areas. Leaf scorching is not a problem in all test crops (maize, lettuce and cabbage) even when overhead irrigation is used.

The major challenge for using AF effluent is its contribution to $\mathrm{N}$ and $\mathrm{P}$ uptake in maize and cabbage crops. The effluent was unfit for maize production with respect to $\mathrm{N}$ and $\mathrm{P}$ concentrations. However, this was different to cabbage, whereby its contribution to $\mathrm{N}$ and $\mathrm{P}$ was acceptable in climatic region 4 in all soils except for coarse sandy soil. It was concluded that the effluent may be diluted to meet the acceptable nutrient concentration required for maize production.

Municipalities are concerned with meeting effluent discharge quality. However, alternatively, agricultural systems can act as sinks for nutrient removal via crop uptake. However, the maize and cabbage rotation showed to be the less effective cropping system to remove nutrients from $\mathrm{AF}$ effluent since more $\mathrm{N}$ and $\mathrm{P}$ are loaded into the soil, where they potentially cause pollution.

Clogging, corrosion and scaling of irrigation equipment are not expected when AF effluent is used. However, although the effluent quality parameters were at least tolerable, it is recommended to consider management practices such as periodic acidification of the irrigation water and installation of a filtration system.

The microbial risks for irrigation with AF effluent depends on the irrigation system and crop type. Microbial contamination risks are not expected in maize irrigated with AF effluent regardless of the irrigation system used. However, lettuce and cabbage are at higher microbial contamination risks when overhead irrigation is used. Therefore, it is recommended to use surface irrigation and further effluent treatment to reduce microbial loads for unrestricted use is also strongly recommended and should be explored.

Author Contributions: Conceptualization, W.M. and A.O.O.; methodology, W.M.; software, W.M.; formal analysis, W.M. and A.O.O.; investigation, W.M.; resources, A.O.O.; data curation, W.M.; writing-original draft preparation, W.M.; writing-review and editing, W.M. and A.O.O.; visualization, W.M.; supervision, A.O.O.; project administration, A.O.O.; funding acquisition, A.O.O. Both authors have read and agreed to the published version of the manuscript.

Funding: This research was funded by the Water Research Commission (WRC), K5/2777.

Institutional Review Board Statement: Not applicable.

Informed Consent Statement: Not applicable.

Data Availability Statement: The data used during this study can be obtained from Musazura [38].

Acknowledgments: The authors acknowledge the Water Research Commission (WRC) K5/2777.

Conflicts of Interest: The authors declare no conflict of interest. 


\section{Appendix A}

Table A1. Analysis of variance table showing mean squares for nutrient (NPK) uptake in different cropping systems, soil types, climatic regions and irrigation types.

\begin{tabular}{|c|c|c|c|c|c|c|}
\hline Source of Variation & D.f. & $\mathbf{N}$ & & $\mathbf{P}$ & & $\mathbf{K}$ \\
\hline Climatic region & 3 & 217,614 & $* * *$ & 1954.6 & $* * *$ & 36,198 \\
\hline Cropping system & 1 & 145,034 & $* *$ & 14,062 & $* * *$ & 1778 \\
\hline Irrigation system & 1 & 1695 & & 98.3 & & 42 \\
\hline Soil type & 2 & 13,290 & & 3183.9 & $* * *$ & 968 \\
\hline Climatic region * Cropping system & 3 & 100,965 & $* * *$ & 1891.1 & $* * *$ & 185 \\
\hline Climatic region * Irrigation system & 3 & 3499 & & 81.2 & & 87 \\
\hline Cropping system * Irrigation system & 1 & 1878 & & 0 & & 210 \\
\hline Climatic region * Soil type & 6 & 6160 & & 443.3 & & 352 \\
\hline Cropping system $*$ Soil type & 2 & 17,984 & & 231.9 & & 125 \\
\hline Irrigation system * Soil type & 2 & 4612 & & 65.7 & & 103 \\
\hline $\begin{array}{c}\text { Climatic region * Cropping system * Irrigation } \\
\text { system }\end{array}$ & 3 & 929 & & 75.4 & & 221 \\
\hline Climatic region ${ }^{*}$ Cropping system $*$ Soil type & 6 & 9584 & & 241.7 & & 103 \\
\hline Climatic region * Irrigation system * Soil type & 6 & 2570 & & 21.6 & & 94 \\
\hline Cropping system * Irrigation system * Soil type & 2 & 316 & & 22.1 & & 59 \\
\hline $\begin{array}{c}\text { Climatic region }{ }^{*} \text { Cropping system * Irrigation } \\
\text { system }{ }^{*} \text { Soil type }\end{array}$ & 6 & 707 & & 31.5 & & 107 \\
\hline Residual & 96 & 13,027 & & 317.7 & & 659 \\
\hline Total & 143 & & & & & \\
\hline
\end{tabular}

\section{References}

1. Parliamentary Monitoring Group. Urbanisation. Available online: https://pmg.org.za/page/Urbanisation (accessed on 11 July 2021).

2. Mokoele, J.; Sebola, M. Unplanned urbanisation in South African cities: The emergence of urban environmental problems. In Proceedings of the 7th International Conference on Business and Economic Development (ICBED), New York, NY, USA, 9-10 April 2018; pp. 574-584.

3. Gutterer, B.; Sasse, L.; Panzerbieter, T.; Reckerzügel, T. Decentralised Wastewater Treatment Systems (DEWATS) and Sanitation in Developing Countries; Water, Engineering and Development Centre (WEDC): Leicestershire, UK, 2009; ISBN 9781843801283.

4. Magwaza, S.T.; Magwaza, L.S.; Odindo, A.O.; Mditshwa, A.; Buckley, C. Evaluating the feasibility of human excreta-derived material for the production of hydroponically grown tomato plants-Part II: Growth and yield. Agric. Water Manag. 2020, 234, 106115. [CrossRef]

5. Bame, I.B.; Hughes, J.C.; Titshall, L.W.; Buckley, C.A. Leachate characteristics as influenced by application of anaerobic baffled reactor effluent to three soils: A soil column study. Chemosphere 2013, 93, 2171-2179. [CrossRef]

6. Busari, T.; Senzanje, A.; Odindo, A.; Buckley, C. Effect of intercropping madumbe [Colocasia esculenta) and rice (Oryza sativa L.) on yield and land productivity under different irrigation water management techniques with effluent water. J. Water SA 2020, 46, 205-212.

7. Bame, I.B.; Hughes, J.C.; Titshall, L.W.; Buckley, C.A. The effect of irrigation with anaerobic baffled reactor effluent on nutrient availability, soil properties and maize growth. Agric. Water Manag. 2014, 134, 50-59. [CrossRef]

8. Abdel-Aziz, R. Impact of Treated Wastewater Irrigation on Soil Chemical Properties and Crop Productivity. Int. J. Water Resour. Arid Environ. 2015, 4, 30-36.

9. Jaramillo, F.M.; Restrepo, I. Wastewater Reuse in Agriculture: A Review about Its Limitations and Benefits. Sustainability 2017, 9, 1734. [CrossRef]

10. Food and Agriculture Organisation. Wastewater Treatment and Use in Agriculture; Volume Irrigation and Drainage Paper 47; FAO, Ed.; FAO: Rome, Italy, 1992; ISBN 92-5-103135-5.

11. Department of Water and Sanitation. South African Water Quality Guidelines, 2nd ed.; DWS (Formerly DWAF): Pretoria, South Africa, 1996; Volume 4, ISBN 0-7988-5342-5.

12. du Plessis, M.; Annandale, J.G.; Benade, N.; Van der Laan, M.; Jooste, S.; du Preez, C.; Barnard, J.; Rodda, N.; Dabrowski, J.; Genthe, B.; et al. Risk Based, Site-Specific, Irrigation Water Quality Guidelines. Volume 1: Description of Decision Support System; Water Research Commission (WRC): Pretoria, South Africa, 2017; ISBN 978-1-4312-0910-1. 
13. Department of Water and Sanitation. Revision of General Authorisations in Terms of Section 39 of the National Water Act. 1998. 2013. Available online: https://www.greencape.co.za/assets/Water-Sector-Desk-Content/DWS-Revision-of-generalauthorisation-in-terms-of-the-National-Water-Act-notice-169-2013.pdf (accessed on 6 September 2021).

14. Food and Agriculture Organisation. Water Quality for Agriculture; FAO, Ed.; FAO: Rome, Italy, 1985; ISBN 92-5-102263-1.

15. World Health Organisation. Guidelines for the Safe Use of Wastewater and Excreta in Agriculture and Aquaculture; WHO: Genever, Switzerland, 1989; ISBN 92-4-152248-9.

16. World Health Organisation. Guidelines for the Safe Use of Wastewater, Excreta and Greywater: Exctreta and Greywater Use in Agricuture; WHO: Geneva, Switzerland, 2006; Volume 4, p. 191. ISBN 92-4-154685-9.

17. United States Environmental Protection Agency. Guidelines for Water Reuse; EPA/600/R-12/618; United States Environmental Protection Agency: Washington, DC, USA, 2012.

18. Fessehazion, M.K.; Annandale, J.G.; Everson, C.S.; Stirzaker, R.J.; Tesfamariam, E.H. Evaluating of soil water balance (SWB-Sci) model for water and nitrogen interactions in pasture: Example using annual ryegrass. Agric. Water Manag. 2014, 146, $238-248$. [CrossRef]

19. Kalra, Y. Handbook of Reference Methods for Plant Analysis; CRC Press: New York, NY, USA, 1997; p. 320. ISBN 9781420049398.

20. Andersson, K.; Rosemarin, A.; Lamizana, B.; Kvarnström, E.; McConville, J.; Seidu, R.; Dickin, S.; Trimmer, C. Sanitation, Wastewater Management and Sustainability from Waste Disposal to Resource Recovery; United Nations Environment Programme and Stockholm Environment Institute: Nairobi, Kenya; Stockholm, Sweden, 2016; ISBN 978-92-807-3488-1.

21. Lal, K.; Minhas, P.S.; Yadav, R.K. Long-term impact of wastewater irrigation and nutrient rates II. Nutrient balance, nitrate leaching and soil properties under peri-urban cropping systems. Agric. Water Manag. 2015, 156, 110-117. [CrossRef]

22. Sharpley, A. Managing agricultural phosphorus to minimize water quality impacts. Sci. Agric. 2016, 73, 1-8. [CrossRef]

23. Conradie, D.C.U. South Africa's Climatic Zones: Today, Tomorrow. In Proceedings of the International Green Building Conference and Exhibition, Sandton, South Africa, 25-26 July 2012.

24. VSN International. Genstat for Windows. Available online: www.Genstat.co.uk (accessed on 3 April 2021).

25. Erel, R.; Eppel, A.; Yermiyahu, U.; Ben-Gal, A.; Levy, G.; Zipori, I.; Schaumann, G.E.; Mayer, O.; Dag, A. Long-term irrigation with reclaimed wastewater: Implications on nutrient management, soil chemistry and olive (Olea europaea L.) performance. Agric. Water Manag. 2019, 213, 324-335. [CrossRef]

26. Echeverría, I.; Machicado, L.; Saavedra, O.; Escalera, R.; Heredia, G.; Montoya, R. Domestic Wastewater Treated by Anaerobic Baffled Reactors and Gravel Filters as a Resource to Be Used in Agriculture. Rev. Investig. Desarro. 2019, 19, 63-72. [CrossRef]

27. Kiziloglu, F.M.; Turan, M.; Sahin, U.; Angin, I.; Anapali, O.; Okuroglu, M. Effects of wastewater irrigation on soil and cabbageplant (brassica olerecea var. capitate cv. yalova-1) chemical properties. J. Plant Nutr. Soil Sci. 2007, 170, 166-172. [CrossRef]

28. Sahin, U.; Ekinci, M.; Ors, S.; Turan, M.; Yildiz, S.; Yildirim, E. Effects of individual and combined effects of salinity and drought on physiological, nutritional and biochemical properties of cabbage (Brassica oleracea var. capitata). Sci. Hortic. 2018, 240, 196-204. [CrossRef]

29. Masa, R.; Khan, Z.; Chowa, G. Youth food insecurity in Ghana and South Africa: Prevalence, socioeconomic correlates, and moderation effect of gender. Child. Youth Serv. Rev. 2020, 116, 105180. [CrossRef]

30. Ungureanu, N.; Vlădut,, V.; Voicu, G. Water Scarcity and Wastewater Reuse in Crop Irrigation. Sustainability 2020, $12,9055$. [CrossRef]

31. Hashem, M.S.; Qi, X. Treated Wastewater Irrigation-A Review. Water 2021, 13, 1527. [CrossRef]

32. FAO. Users Manual for Irrigation with Treated Wastewater; TC/D/Y5009F/1/10.03/100; Food and Agriculture Organisation Regional Office near East: Cairo, Egypt, 2003.

33. Musazura, W.; Odindo, A.O.; Tesfamariam, E.H.; Hughes, J.C.; Buckley, C.A. Nitrogen and phosphorus fluxes in three soils fertigated with decentralised wastewater treatment effluent to field capacity. J. Water Reuse Desalination 2019, 9, 142-151. [CrossRef]

34. Levy, G.; Fine, P.; Bar-Tal, A. Treated Wastewater in Agriculture: Use and Impacts on the Soil Environments and Crops; Wiley: New York, NY, USA, 2011; p. 464. ISBN 9781444322514.

35. Dirwai, T.L.; Senzanje, A.; Mabhaudhi, T.; Buckley, C.A. Moistube irrigation fouling due to anaerobic filtered effluent (AF) and horizontal flow constructed wetland (HFCW) effluent. Sci. Rep. 2021, 11, 7124. [CrossRef]

36. Farhadkhani, M.; Nikaeen, M.; Yadegarfar, G.; Hatamzadeh, M.; Pourmohammadbagher, H.; Sahbaei, Z.; Rahmani, H.R. Effects of irrigation with secondary treated wastewater on physicochemical and microbial properties of soil and produce safety in a semi-arid area. Water Res. 2018, 144, 356-364. [CrossRef]

37. De Sanctis, M.; Del Moro, G.; Levantesi, C.; Luprano, M.L.; Di Iaconi, C. Integration of an innovative biological treatment with physical or chemical disinfection for wastewater reuse. Sci. Total Environ. 2016, 543, 206-213. [CrossRef]

38. Musazura, W. Suitability of the Decentralised Wastewater Treatment effluent for agricultural use: Decision Support System approach. Mendeley Data 2021. [CrossRef] 